\title{
THERMAL WAVE METHODS: SOME EXPERIMENTAL PITFALLS.
}

\author{
M. WÜBBENHORST and J. VAN TURNHOUT*
}

Dept. of Polymer Technology, Delft University of Technology, P.O. Box 5045, 2600 GA Delft, The Netheriands.

* TNO - Plastics and Rubber Research Institute, P.O. Box 6031, 2600 JA Delft, The

Netherlands

\begin{abstract}
Uncertainties in the spatial distributions calculated from thermal wave data are normally attributed to the ill-posed deconvolution problem. This paper focusses on the impact of "ill-posed" experimental conditions on the pyroelectric spectra. The spectra are affected by structural inhomogeneities and temporal changes in the electrical and thermal properties, by distortions caused by the electric circuit, by frequency-dependent properties and by piezoelectricity. Experimental results are given for PVDF and $\mathrm{LiTaO}_{3}$. Suggestions are made to reduce the effect of experimental errors.
\end{abstract}

\section{Introduction}

Polarization and space charge distributions in electret films are frequently determined by means of thermal wave techniques rather than with the more elaborated piezoelectrically-based acoustical techniques. The thermal techniques apply either heat pulses [1] or continuous waves [2] and are attractive because of their moderately priced equipment and their high spatial resolution. Since the transient and $\mathrm{cw}$ technique are basically equivalent, we restrict ourself to the laser-intensity modulated, cw-method (LIMM) developed by Lang and Das-Gupta [2].

LIMM has undergone gradual improvements regarding reliability and achievable resolution owing to the use of advanced numerical deconvolution procedures (singular value decomposition, regularization techniques, etc. [3-5]). The prominent features of LIMM have been demonstrated for simulated and for measured data $[3,6,7]$. The reproducibility of nearly homogeneously polarized samples is always satisfying, whereas for nonhomogeneous distributions the predictions and the outcome of LIMM is less satisfactory.

In order to obtain well-defined nonuniform properties we have used two types of model samples; one consisted of a bimorph of oppositely poled PVDF films, the other was made from poled PVDF and of $\mathrm{LiTaO}_{3}$ both provided with a thick paint layer. Their pyroresponse allowed us to obtain a quantitative idea of the impact of possible errors on LIMM spectra.

\section{Overview of effects on LIMM-spectra}

2.1 Limitations of the electrical and thermal model

For a pyroelectric film of thickness $\mathrm{L}$ and area $\mathrm{A}$, containing a nonuniform polarization $\bar{P}+P(x)$ and a space charge distribution $\rho(x)$, the LIMM-current $I_{-}(\omega)$ reads [3]: 


$$
\begin{aligned}
I_{\sim}(\omega)=\alpha_{p} A \bar{P} \frac{d \bar{T}(\omega)}{d t}+ & \frac{A}{L}\left(\alpha_{p}+\alpha_{L}-\alpha_{\epsilon}\right) \int_{Q^{L}}^{L} P(x) \frac{\partial T(x, \omega)}{\partial t} d x+ \\
& \left.+\frac{A}{L}\left(\alpha_{L}-\alpha_{\epsilon}\right) \int_{0}^{x} \rho(x) \int_{0}^{x} \frac{\partial T\left(x^{\prime}, \omega\right)}{\partial t}-\frac{d \bar{T}(\omega)}{d t}\right] d x^{\prime} d x
\end{aligned}
$$

where $\alpha_{p}, \alpha_{L}$ and $\alpha_{\varepsilon}$ denote the temperature coefficients of the polarization, the thickness and the permittivity, respectively. For convenience we have considered only the 1 st and 2nd term due to the permanent polarisation $\mathrm{P}+\mathrm{P}(\mathrm{x})$ in our model calculations. Eq. 1 can easily be extended to multi-layers provided that each layer is homogeneous.

On the other hand an $x$-dependent thermal expansion $\alpha_{L}(x)$, permittivity $\varepsilon(x)$ or $\alpha_{b}(x)$ require a more general expression given in [8]. The consideration of position-dependent properties is a prerequisite in the analysis of LIMM-spectra of multilayers, such as laminates of different polymers, coatings on pyroelectric sensors etc.. Such an analysis is still straightforward if all layers have space-independent properties. However, a LLMManalysis is not feasible if the material properties are spatially distributed in an unknown way.

Structural gradients in properties can e.g. be caused by differences in the degree of crystallinity. As a consequence, dielectric or thermal anisotropies appear near the surface, where LIMM possesses its highest resolution.

\subsection{Frequency-dependent dielectric properties}

The permittivity will affect the pyroelectric spectrum of multi-layers if each layer has different frequency-dependent dielectric constants $\varepsilon_{i}(\omega)$. But, also LIMM-measurements on monolayers might suffer from dielectric relaxations, via the term $\alpha_{\varepsilon}(\omega, T)=$ $\operatorname{din} \varepsilon^{\prime}(\omega, T) / d T$. This term can be related to the dielectric losses by the approximation [9]

$$
\alpha_{\epsilon}(\omega, T)=\frac{\partial \ln \epsilon^{\prime}(\omega, T)}{\partial T} \approx \frac{2 E_{A}}{\pi R T^{2}} \cdot \frac{\epsilon^{\prime \prime}(\omega, T)}{\epsilon^{\prime}(\omega, T)}
$$

where $E_{A}$ is the activation energy at the temperature $T$, and $\varepsilon^{\prime}, \varepsilon^{\prime \prime}$ and $R$ denote the real and imaginary part of the permittivity and the gas-constant respectively. Strong relaxation processes - like the glass transition - may lead to rather high values $\left(>10^{-2}\right)$ of the term $\alpha_{\mathrm{s}}$ (cf. Fig.3).

\subsection{Bending and tensile piezoelectricity}

The one-sided LIMM-heating causes an asymmetric expansion, which in a free film results in a deformation. In films having an inhomogeneous polarization this deformation causes in return a piezo-electric response $[10,11]$. Bending resonances (flexural waves) are visible in many published LIMM-spectra. The fundamental frequency $f_{f}$ depends on the film width $b$, the thickness $L$ and the transverse sound velocity $v_{t}$ and occurs in PVDFfilms at around $1 \mathrm{kHz}$ :

$$
f_{x}=\frac{9}{32} \frac{\pi L v_{t}}{b^{2}}
$$


The thermally generated deformations give also rise to a nonresonant piezo-contribution in PVDF, which might reach $20 \%$ of the pyro-current at high frequencies. The dimensions, the elastic properties and the way of fixing the pyroelectric sample are therefore all essential.

\subsection{Characteristics of the electronic circuit}

The electronics of a LDMM-set-up includes 3 main components:

i) modulation driver (e.g. for an acousto-optic-modulator)

ii) preamplifier, usually a current- to- voltage converter, and

iii) gain-phase analyzer, e.g. lock-in amplifier or frequency-response analyzer.

The errors resulting from the electronic parts arise from the limited bandwidth of the gain/phase measurement, noise and interference between driving voltage and input circuit. All these error sources become the more important the higher the operation frequency $(>1 \mathrm{kHz})$.

The gain/phase characteristics of the preamplifier often form the crucial factor. They depend on the gain, filter time constants, capacitive load, and modulation level. Our lock-in amplifier on the other hand had an excellent gain/phase behaviour throughout the whole frequency range from $0.001 \mathrm{~Hz}$ to $100 \mathrm{kHz}$.

We have corrected all the LIMM-spectra measured by performing a calibration with a fast fotodiode (cut-off frequency $100 \mathrm{MHz}$, capacitance $<10 \mathrm{pF}$ ); for each sample such a correction is crucial.

\subsection{Temporal influences}

Drift of the laser intensity, degradation of the heat absorbing layer or temporal changes in the polarization due to depolarization processes or charge relaxations in PVDFfilms may also affect the LIMM-spectrum. One way to visualize temporal effects is the use of a random frequency scan rather than a sequential one.

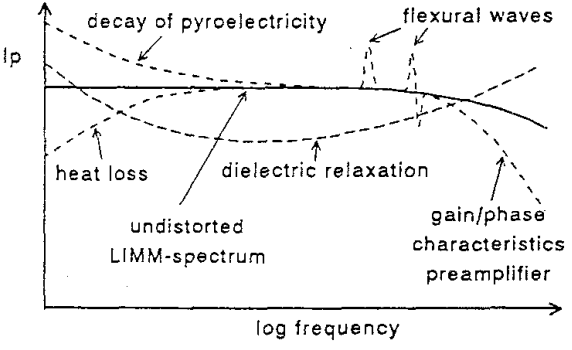

Fig.1 Survey of possible distortions of a LIMM-spectrum.

A summary of the impact of the various processes that can alter the LIMM-spectrum is given in Fig.1.

\section{Experiments}

3.1 Experimental set-up

The equipment used for LIMM is described in detail in [12]. A He-Ne-laser combined with an acousto-optic modulator provides the intensity modulated radiation $(<5 \mathrm{~mW}$ r.m.s). The sample is placed in a holder either under atmospheric pressure or in vacuum. The pyroelectric current was converted by a current amplifier (Keithley, model 428) to an ac-voltage, and was analyzed by a digital signal processing lock-in amplifier (Stanford Research System, SR850).

The dielectric measurements were performed over a wide frequency range $\left(10^{-3} \mathrm{~Hz}\right.$ 
to $10^{6} \mathrm{~Hz}$ ) by means of an LCR-meter (HP 4284A) and a low-frequency system consisting of an electrometer (TNO) and a frequency response analyzer (Schlumberger, model 1260). Films of PVDF were investigated from -150 to $250^{\circ} \mathrm{C}$ in a nitrogen-gas heated cryostat.

The device programming and data acquisition were computer controlled.

\subsection{Dielectric studies on PVDF-films}

Dielectric measurements were performed on 2 biaxially stretched PVDF films

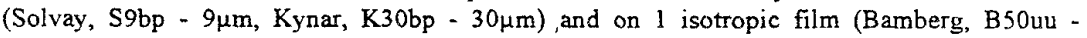
$50 \mu \mathrm{m})$. The real part of the permittivity for isotropic PVDF is given in Fig.2.

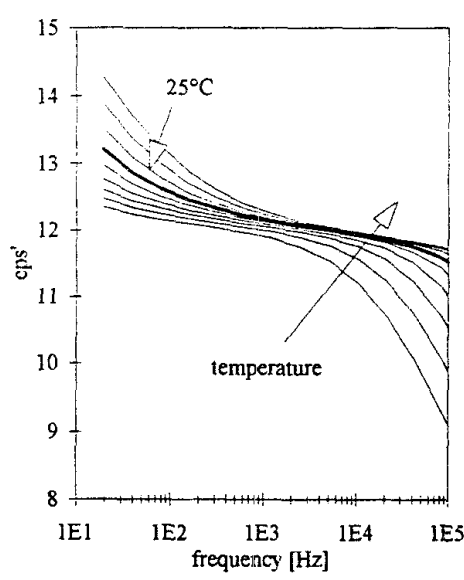

Fig.2 Frequency dependence of $\varepsilon^{\prime}$ of isotropic PVDF (>90\% $\alpha$-phase) at various temperatures around $25^{\circ} \mathrm{C}(0,5$, .. $40^{\circ} \mathrm{C}$ ).

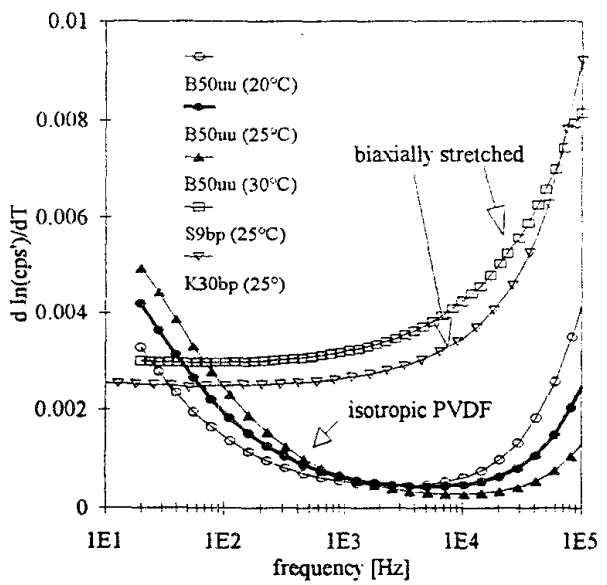

Fig. 3 Frequency dependence of $\alpha_{\mathrm{c}}=$ dine'/dT for 2 poled, stretched PVDF films at $25^{\circ} \mathrm{C}$. In addition, the influence of the temperature $\left(20\right.$ to $\left.30^{\circ} \mathrm{C}\right)$ on $\alpha_{c}$ is shown for isotropic PVDF.

Fig.3 shows that the frequency dependence of the temperature coefficient $\alpha_{\mathrm{c}}$ differs markedly for the biaxially stretched and the isotropic PVDF films. The high frequency upswing in $\alpha_{2}$ is due to the influence of the glass-transition, while the low-frequency increase for isotropic PVDF is caused by the mid-temperature relaxation of the $\alpha$-phase.

To illustrate the effect of a frequency-dependent $\alpha_{c}$ on the LIMM-spectrum of a nonuniformly polarized sample we have calculated the pyro-response of a bimorph of oppositely poled PVDF of $9 \mu \mathrm{m}$ with eqn.(1) using the $\alpha_{a}$-data from Fig. 3 and the parameters $\alpha_{\mathrm{L}}=2 \cdot 10^{-4} \mathrm{~K}^{-1}$ and $\alpha_{\mathrm{p}}=-4 \cdot 10^{-4} \mathrm{~K}^{-1}$.

We see that up to $4 \cdot 10^{3} \mathrm{~Hz}$ biaxially stretched PVDF is scarcely affected by dielectric relaxations, whereas the LIMM spectrum of isotropic PVDF clearly is.

\subsection{Piezoelectric effects}

To estimate the contribution of piezoelectricity to the LIMM-response we have investigated mono- and bilayers of $\mathrm{PVDF}$ and $\mathrm{LiTaO}_{3}$. 
Fig.5 shows the LIMM-spectrum of $20 \times 10 \mathrm{~mm}^{2}$ biaxially stretched PVDF-film (Solvay, L=40 $\mathrm{m}$ ) for different mechanical fixations. The samples were coated with a $20 \mu \mathrm{m}$ thick black paint which served both as heat absorber and as electrically neutral layer.

Surprisingly, no curve approachs the expected zero current level at high frequencies, when the thermal waves cannot reach the pyroactive PVDF-layer anymore. Obviously, this highfrequency response must originate from a thermoelastically generated (negative) piezocurrent.

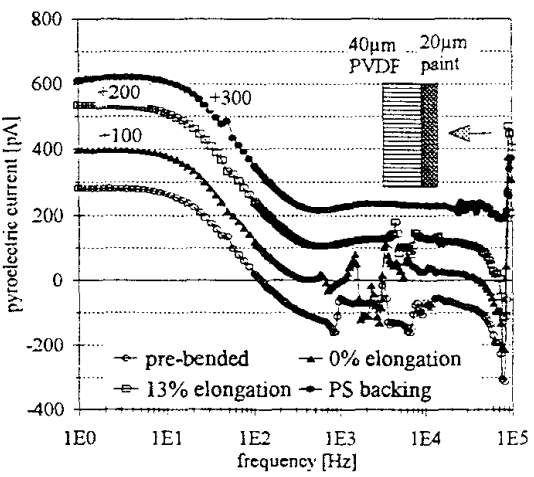

Fig.5 LIMM-spectra of PVDF coated with a $20 \mu \mathrm{m}$ black paint in order to reveal the contribution of piezoelectricity. Results for different mechanical fixations are shown: prebended, $0 \%$ strain, $13 \%$ strain and backed with PS- foam.

For more clearity, the curves have been displayed by 100,200 , and 300 units. The lowest curve was measured on a loose, pre-bended, sample. This shows strong resonances above $1 \mathrm{kHz}$. The intermediate curves correspond to samples clamped taut under 0 and $13 \%$ strain. The resonances are hardly suppressed. However, backing the PVDF with polystyrene foam does suppress the resonances (upper curve) effectively.

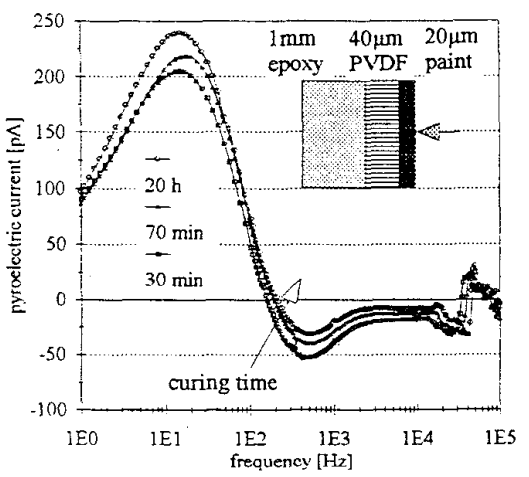

Fig.6 LIMM-spectra of the same PVDF sample, but now coated with 1 mm epoxy on the back side as a function of the degree of cure.

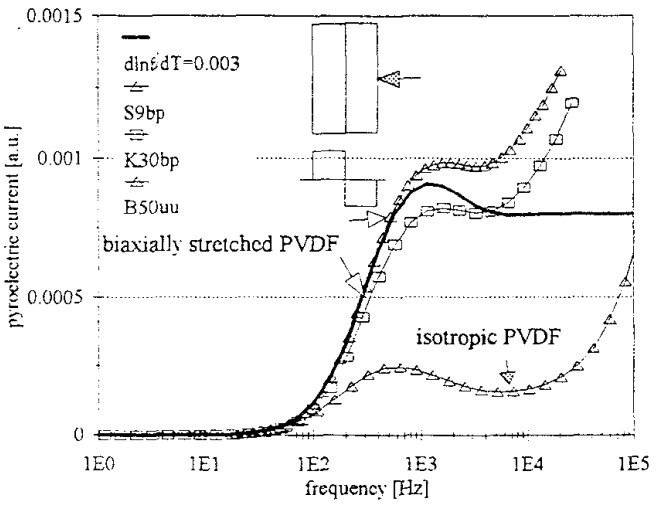

Fig.4 Calculated LIMM-spectra for a bimorph consising of oppositely poled PVDF films. The solid curve (o a constant $\alpha_{a}=3 \cdot 10^{-3} \mathrm{~K}^{-1}$, while the other curves are calculated using the actual $\alpha_{c}$-data of Fig. 3. 
Both the bending and (high frequency) tensile contribution can be reduced by applying a thick layer of epoxy, cf. Fig.6. The higher the extent of cure of the resin the lower the remaining high-frequency piezo-contribution. However, such a solid backing introduces unwanted heat losses, which tesult in a lower pyrocurrent at low frequencies.

The LIMM-data of Li$\mathrm{TaO}_{3}$ in Fig.7 dernonstrate that $\mathrm{LiTaO}_{3}$ is more suitable in model-systems than PVDF, because it is fully crystalline and is much stiffer. Accordingly, hardly any piezoelectric contribution can be noticed in Fig. 7.

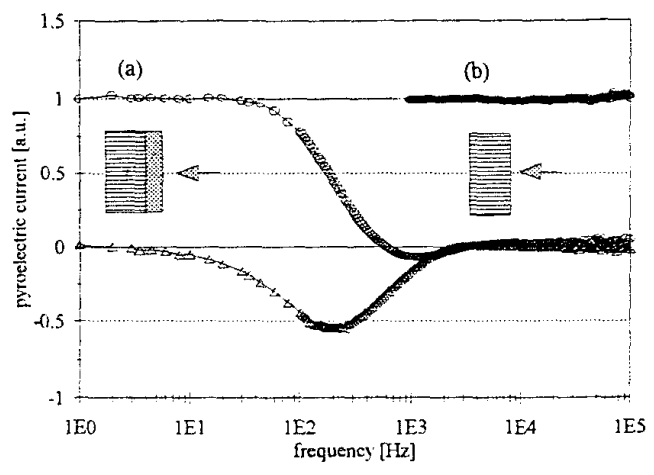

Fig.7 Pyroelectric spectra of $75 \mu \mathrm{m} \mathrm{LiTaO}$ a) with a $20 \mu$ m paint coating and $b$ ) without. For a) both the in-phase and out-phase currents are shown.

\section{Conclusions and recommendations}

LIMM-experiments on polymer films may suffer from several experimental errors, in particular when the properties are nonuniform or the samples are asymmetric. However, most errors can be corrected, e.g. by applying a calibration or by carefully checking the validity of the electrical or thermal model.

Two mechanisms should be addressed particularly, piezoelectricity and dielectric dispersion. The first can be suppressed with a (thermally insulating) backing, by taking symmetric samples (e.g. bimorphs) or by illuminating on two sides. The dielectric dispersion should be measured separately. It can then be accounted for by using a generalization of eqn.1.

\section{REFERENCES}

[1] R.E. Collins, Rev. Sci. Instrum. 48,83 (1977).

[2] S.B. Lang and D.K. Das-Gupta, Ferroelectrics, 60,23 (1984)

[3] S.B. Lang, Ferroelectrics, 118,343 (1991).

[4] N. Link, S. Bauer, B. Ploss, J. Appl. Phys. $\underline{69}, 2759$ (1991).

[5] M. Wübbenhorst, Ph.D. thesis, university Leipzig, (1989).

[6] B. Ploss, R. Emmerich, S. Bauer, J.Appl. Phys., 72,5363 (1992).

[7] M. Wübbenhorst, P. Wünsche, Prog. Coll. Polym. Sci., 85, 23 (1991)

[8] Y. Wada, R. Hayakawa, Japan. J. Appl. Phys., 15, 2041 (1976).

[9] P.A.M. Steeman, J. van Turnhout, submitted to Macromolecules, (1994).

[10] H. Sussner, K. Dransfeid, J, Polym. Sci.: Polym. Phys. Ed., 16, 529 (1978).

[11] E. Fukada, G.M. Sessler, J.E. West, A. Berraissoul, P.Günther, J. Appl. Phys., 62 3643 (1987).

[12] M. Wübbenhorst, J. van Turnhout, L. Alili, Ferroelectrics (1994), in press. 